\title{
Giant Faraday rotation in mesogenic organic
}

\section{molecules}

Stefaan Vandendriessche, ${ }^{*, \dagger}$ Stijn Van Cleuvenbergen, ${ }^{\dagger}$ Pieter Willot, ${ }^{\ddagger}$ Gunther Hennrich, ${ }^{\mathbb{I}}$ Monika Srebro, ${ }^{\S}$ Ventsislav K. Valev, $\|$ Guy Koeckelberghs, ${ }^{\ddagger}$ Koen Clays, ${ }^{\dagger}$ Jochen Autschbach, $^{\perp}$ and Thierry Verbiest ${ }^{\dagger}$

Molecular Electronics and Photonics, 3001 Heverlee, Belgium, Polymer Chemistry and

Materials, Departamento de Quimica Organica, Universidad Autonoma de Madrid, Cantoblanco, Madrid 28049, Spain, Department of Theoretical Chemistry, Faculty of Chemistry, Jagiellonian University, 30-060 Krakow, Poland, Nanophotonics Centre, Cavendish Laboratory University of Cambridge

Cambridge, CB3 OHE

United Kingdom, and Department of Chemistry

University at Buffalo

State University of New York

Buffalo, NY 14260-3000

E-mail: stefaan.vandendriessche@chem.kuleuven.be

\footnotetext{
*To whom correspondence should be addressed

$\dagger$ KU Leuven

${ }^{\ddagger}$ KU Leuven

IIUniversidad Autonoma de Madrid

$\S$ Jagiellonian University

Cambridge

${ }^{\perp}$ Buffalo
}

\section{Abstract}


Faraday rotation, the rotation of the polarization of light due to a magnetic field in the direction of propagation of the light, is used in applications ranging from quantum memory to the detection of biomagnetic fields. For these applications large Faraday rotation is necessary, but absorption of light is detrimental. In search of these properties, we have characterized the Verdet constant of a so far unexplored class of mesogenic organic molecules. We report their spectra and provide an interpretation. A Verdet constant of almost $2.5 \cdot 10^{5} \operatorname{deg} \mathrm{T}^{-1} \mathrm{~m}^{-1}$ is found around $520 \mathrm{~nm}$. This Verdet constant is three orders of magnitude larger than the largest known for organic molecules in a region without spectral features. We attribute this enormous Faraday rotation to resonant enhancement by a triplet excitation which does not appear in the linear absorption spectrum, and near-resonant enhancement by low-energy singlet excitations. Furthermore we are able to switch the Faraday rotation by changing the liquid crystal phase of the compound. These results demonstrate a new class of Faraday rotating materials with great potential to replace current materials and improve existing applications. The inherent flexibility in the synthesis of this class of molecules opens a new field of research in Faraday rotation.

keywords: Faraday rotation, liquid crystal, Verdet constant, TD-DFT calculations

\section{Introduction}

Faraday rotation is the rotation of the plane of polarization of light due to magnetically induced circular birefringence. ${ }^{1}$ Discovered almost two centuries ago, it is now used in a wide range of applications. Faraday rotation is important for optical isolators, ${ }^{2}$ magnetic field sensors, ${ }^{3}$ current sensors, ${ }^{4}$ displacement sensors, ${ }^{5}$ integrated communication optics, ${ }^{6}$ detection of biomagnetic fields, ${ }^{7}$ quantum memory ${ }^{8}$ and many others. It can be described by

$$
\theta=V B L
$$

where $\theta$ is the polarization rotation, $V$ is the Verdet constant, $B$ is the magnetic field parallel to the propagation of light, and $L$ is the length of propagation through the magnetic field. 
The Verdet constant is a wavelength dependent material parameter. Typical Verdet constants of materials currently in use, such as iron oxide films ${ }^{9}$ or substituted iron garnet films, ${ }^{10}$ can reach millions of degrees per tesla per meter in the visible frequency region. However, these paramagnetic materials suffer from the drawback of magnetic saturation in magnetic fields smaller than 1 tesla $^{11}$ and a large temperature dependence. ${ }^{12}$ Organic, diamagnetic materials in contrast saturate at far larger magnetic fields and additionally are relatively insensitive to temperature changes, which is beneficial for sensing applications. An additional advantage of organic materials over inorganic garnets for Faraday rotation are their processability and flexibility. ${ }^{13}$ Unfortunately, diamagnetic organic molecules have relatively small Verdet constants in regions far from resonance. Typical Verdet constants are on the order of hundreds of degrees per tesla per meter in the visible wavelength region, displaying a continuous decline towards longer wavelengths. ${ }^{14-18}$ Only for the larger polythiophenes in specific conditions have larger Verdet constants been reported, on the order of $10^{4} \mathrm{deg} \mathrm{T}^{-1} \mathrm{~m}^{-1} .{ }^{19}$ However, these large Verdet constants currently lack a conclusive explanation.

In search of large Verdet constants without an associated absorption, we have characterized the Verdet constant of an unexplored class of organic molecules. A band in Faraday rotation is

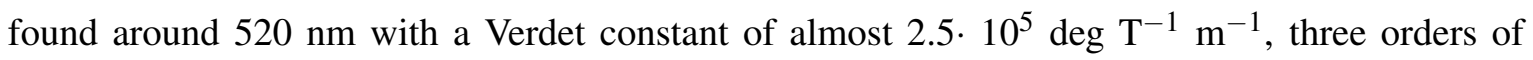
magnitude larger than the largest known for an organic molecule in a region without spectral features. We attribute this giant Faraday rotation to resonant enhancement by weakly absorbing triplet states ${ }^{20-23}$ which are calculated to be present in this region, as well as near-resonant enhancement due to strongly absorbing singlet states at wavelength not much shorter than the wavelength window around $520 \mathrm{~nm}$. The lack of a corresponding strong absorption around $520 \mathrm{~nm}$ is a great advantage for applications.

We are also able to switch the Faraday rotation by changing the particular phase of the compound. The feature in Faraday rotation is present in the crystalline state under $60^{\circ} \mathrm{C}$, but disappears entirely in the isotropic liquid above $130^{\circ} \mathrm{C}$.

These results demonstrate a new class of Faraday rotating materials with great potential to 
replace current materials and improve existing applications. The inherent flexibility in the synthesis of this class of molecules opens a new field of materials for Faraday rotation.

\section{Experimental}

TTB (1,3,5-tris[(4-nitrophenyl)ethynyl]-2,4,6-tris(n-decyloxy)benzene), TTB* (1,3,5-tris[(S)-3,7dimethyloctyloxy]-2,4,6-tris[(4-nitrophenyl)ethynyl]benzene) and BTB*(1,3-bis[(S)-3,7-dimethyloctyloxy)]5-fluoro-2,4,6-tris[(4-nitrophenyl)ethynyl]-benzene) were synthesized as described by Hennrich et al. ${ }^{24,25}$ Thin film samples were created by heating a small amount of material to $120^{\circ} \mathrm{C}$ and allowing this material to spread in between two glass plates through capillary action. The resulting transparent samples were of sufficient optical quality for Faraday rotation measurements.

Spectral Faraday rotation measurements were performed using a 150W Xenon lamp followed by a monochromator. After being linearly polarized, the beam passes through a photo-elastic modulator (Hinds I/FS50) at 45 degrees, operating at $50 \mathrm{kHz}$. Subsequently this beam passes through the sample in a DC magnet, where Faraday rotation occurs. Finally the light passes through an analyzer placed at 90 degrees with respect to the first polarizer. The beam then hits a photomultuplier tube, and the resulting signal is analyzed by a lock-in amplifier (SR830) at a detection frequency of $100 \mathrm{kHz}$. This signal can be correlated to the optical rotation, and is measured for a discrete set of magnetic field strengths, with the Verdet constant determined from the slope of these values. We also measured Faraday rotation with a $632.8 \mathrm{~nm}$ Helium Neon laser and an alternating current magnetic field at $857 \mathrm{~Hz}$ as described by Valev et al. ${ }^{26}$

To assist the interpretation of the experimental findings we performed density functional theory (DFT) computations with the Turbomole package (version 5.727,28). Taking into account the rather large size of the molecules studied experimentally, the aliphatic tails were replaced by methyl groups to reduce the computational cost. As demonstrated by a comparison between the experimental Faraday rotation spectra of TTB and $\mathrm{TTB}^{*}$, the hydrocarbon chains appear to influence a location of particular bands in the spectrum, but they do not change their general characteristics 
(vide infra). Accordingly the hydrocarbon chains are unlikely responsible for the characteristic main features observed for the Faraday rotation. Geometry optimizations employed the BP functional $^{29-31}$ with a standard Turbomole triple- $\zeta$ polarized (TZVP) Gaussian-type basis set. ${ }^{32}$ Calculations of the excitation spectra were performed using time-dependent DFT (TD-DFT) with the B3LYP functional ${ }^{33-35}$ and the TZVP basis set. As pointed out by Peach et al., TD-DFT calculations may significantly underestimate triplet excitation energies due to an underlying triplet instability of the ground state. ${ }^{36}$ In a Hartree-Fock (HF) calculation, the singlet ground state of the TTB model system is indeed confirmed to have a triplet instability. Peach et al. found that the use of the Tamm-Dancoff approximation (TDA) ${ }^{37,38}$ eliminates much of the problem and provides more accurate triplet excitations. A comparison of the full TD-DFT and TD-DFT/TDA results for our samples shows the expected behavior, namely a sizable blue-shift of the triplet excitations, in particular, when the TDA is applied. The effect is more pronounced with a hybrid functional with a larger fraction of exact exchange (e.g. BHLYP vs. B3LYP, see Figure 2 (vide infra) and the SI).

\section{Results and discussion}

In the experimental Faraday rotation spectrum of TTB a peak with a Verdet constant of almost 2.5 . $10^{5} \mathrm{deg} \mathrm{T}^{-1} \mathrm{~m}^{-1}$ is seen centered around $520 \mathrm{~nm}$ (Figure 1). The feature resembles an anomalous dispersion pattern of a refractive property such as the dipole polarizability around an electronic resonance, with a peak followed by a trough when going from longer to shorter wavelengths, superimposed onto a normal dispersion created by intense absorptions at higher energy (wavelengths shorter than $500 \mathrm{~nm}$ ). In addition, strong Faraday rotation is observed below $450 \mathrm{~nm}$ due to resonant enhancement by absorption at these wavelengths. In the absorption spectrum on the other hand, only one band is observed with a center wavelength shorter than $400 \mathrm{~nm}$, with no peaks past $500 \mathrm{~nm}^{24}$

It is necessary to verify first that the observed feature is indeed caused by Faraday rotation. Faraday rotation is linear as a function of the applied magnetic field (Eq. (1)). The optical rotation 


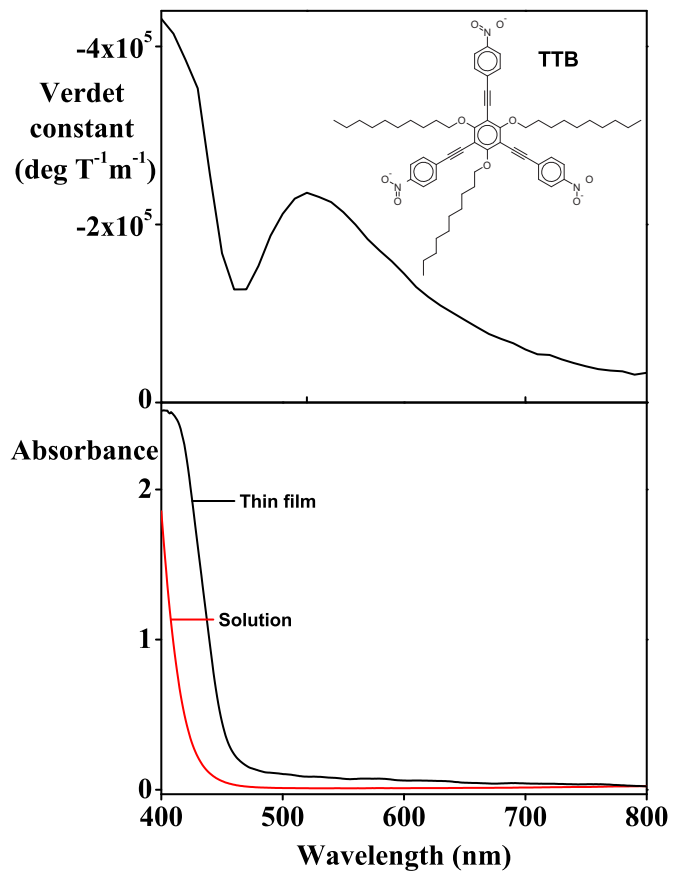

Figure 1: In the experimental Faraday rotation spectrum of a $1.8 \mu \mathrm{m}$ film of TTB (top) a peak is seen at at $520 \mathrm{~nm}$, associated with an anomalous dispersion feature attributed to a triplet excited state near this wavelength. The transition to the triplet excited state is not visible in the absorption spectrum in thin film (bottom, black) or solution (bottom, red) because it is formally spin-forbidden. 
measured in a DC magnetic field is also linear as a function of the magnetic field $\left(R^{2}>0.99\right)$, with no saturation being visible until at least 1 tesla. Despite this, it is not possible to exclude the magnetic alignment of crystallites as a source of optical rotation. To support Faraday rotation as the source of the observed magnetic field dependent optical rotation, we also performed Faraday rotation measurements in an alternating current magnetic field. In these measurements, the same Faraday rotation was observed. The high modulation frequency of the magnetic field allows us to exclude a magnetic alignment as the cause of the observed magnetic field dependent optical rotation. We can conclude that the feature in the observed optical activity is indeed Faraday rotation.

The Verdet constant is a molecular property that is defined by three pertubations: ${ }^{39-41}$ two frequency-dependent electric fields and a static magnetic field. Resonant enhancement may occur via strongly or weakly electric-dipole allowed transition, and potentially also via magnetic-dipole allowed but electric-dipole forbidden transitions. A corresponding band is not visible in the linear absorption spectrum of the molecule, indicating the lack of any significant electric-dipole allowed transition. This excludes the possibility of resonant enhancement by an intermolecular chargetransfer excitation induced by aggregation. Nonetheless, formally electric-dipole forbidden transitions with weak intensity such as singlet-triplet transitions can also resonantly enhance Faraday rotation. ${ }^{20-23}$ Computations and careful experiments on related threefold symmetrical molecules indicate the presence of triplet states close to these energies. ${ }^{42}$

We calculated the electronic states of TTB for the propeller geometry adopted in crystals ${ }^{43}$ (Figure 2). Triplet excitations with a large spin-free component of the transition dipole are present around $527 \mathrm{~nm}$ (see SI), corresponding to the region where the Faraday rotation is observed. While this excitation is formally spin-forbidden, and as such not visible in the absorption spectrum, it may resonantly enhance the Faraday rotation. No electric-dipole forbidden but magnetic-dipole allowed singlet transition was calculated at wavelengths longer than $450 \mathrm{~nm}$ which would therefore exclude a mechanism via resonance with electric-dipole or magnetically allowed singlet transition in the wavelength window of interest.

To shed more light on the hypothesis of a resonant enhancement by a weakly absorbing triplet 


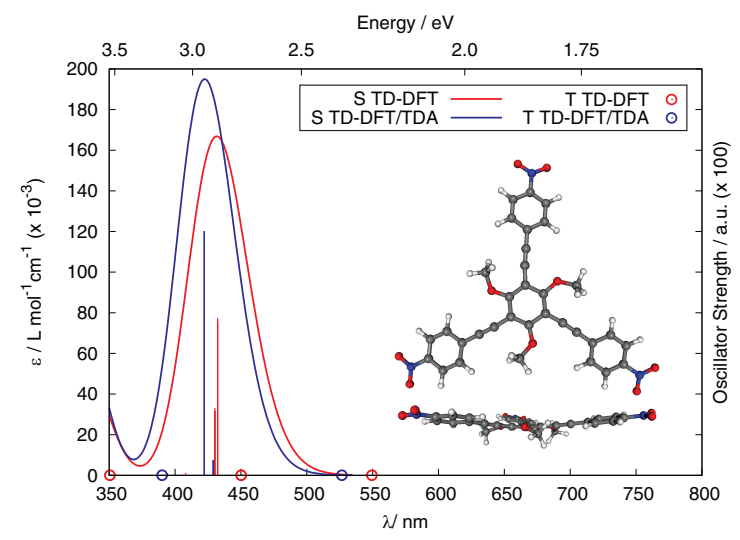

Figure 2: TD-DFT B3LYP/TZVP calculations on the optimized model TTB geometry reveal a triplet excitation at $527 \mathrm{~nm}$, corresponding to the region where the Faraday rotation is observed. S / T stands for singlet / triplet excitation energies calculations.

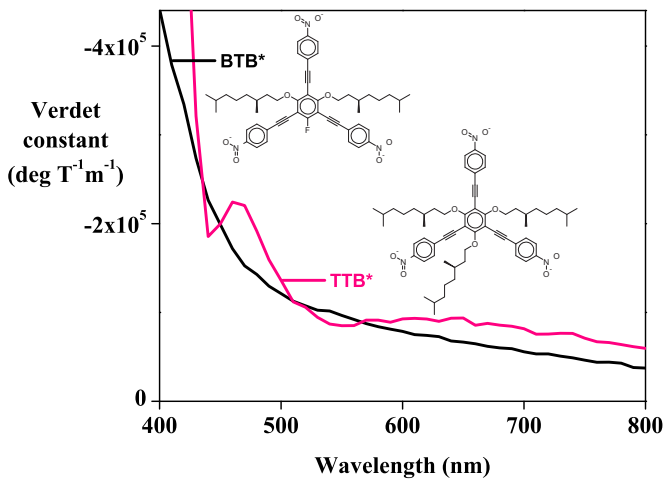

Figure 3: The feature in Faraday rotation in thin films of TTB* is blue-shifted and narrower than in thin films of TTB (Figure 1), due to the bulkiness of the branched alkyl chains in TTB*. Thin films of BTB* however show no separate feature in the Faraday rotation spectrum, despite presence of calculated triplet excitations around $510 \mathrm{~nm}$. The corresponding band is likely hidden in the dispersion due to strongly absorbing singlet states. 
state, we examined two structurally related molecules. TTB* has a blue-shifted and narrower feature in Faraday rotation (Figure 3) than TTB (Figure 1). The bulkiness of the branched alkyl chains impedes a close packing of molecular units but guarantees for a fixed propeller confirmation on the molecular level and a helical twist in the resulting bulk aggregates. This is reflected in a blue-shifted triplet state and the corresponding feature in Faraday rotation. ${ }^{44} \mathrm{TTB} *$ forms a chiral nematic discotic phase between 66 and $99^{\circ} \mathrm{C}$ as a consequence of the of the homochiral nature of the three alkoxy chains. For BTB*, no separate feature in the Faraday rotation spectrum was detected (Figure 3). The calculations performed for a model of BTB* (see SI) reveal, however, a presence of triplet excitations around $510 \mathrm{~nm}$. Taking into account the spectral blue-shift observed in the calculation for BTB* as compared to TTB (which is expected to be even more pronounced when considering branched hydrocarbon chains in the molecule, based on the discussion above) it seems possible that in this case the feature in the Faraday rotation spectrum assigned to resonance with a triplet state is simply hidden in the resonant enhancement by strongly absorbing states. Consequently, one broad band in Faraday rotation spectrum is observed here.

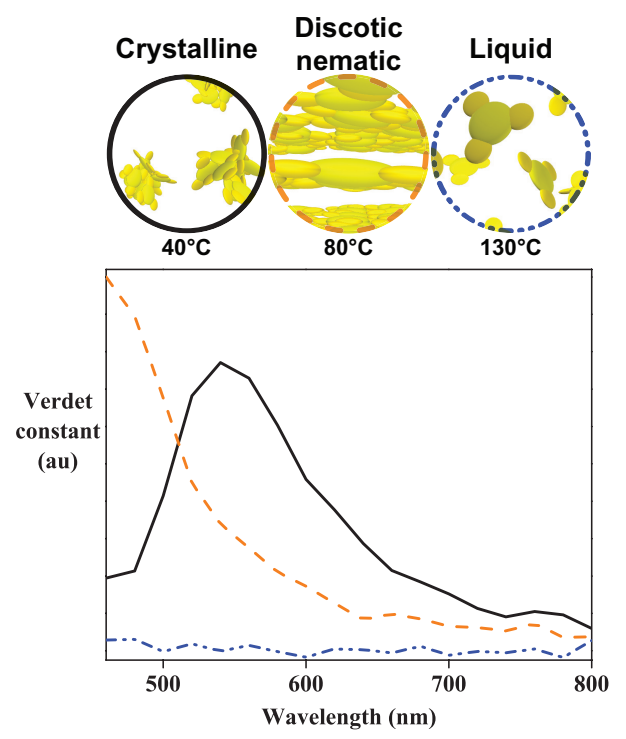

Figure 4: The Faraday rotation spectrum of TTB changes upon heating. The phase change from crystalline (full black) over discotic nematic (striped orange) to an isotropic liquid (dotted blue) is accompanied by a change in conformation and conformational flexibility. Correspondingly, the feature in Faraday rotation is no longer visible in the isotropic liquid phase. 
The liquid crystal nature of the molecule leads us to investigate the effect of temperature on the Faraday rotation spectrum (Figure 4). Under $60^{\circ} \mathrm{C} \mathrm{TTB}$ is crystalline, and it is columnar hexagonal from $60^{\circ} \mathrm{C}$ to $68.5^{\circ} \mathrm{C}$. It displays a discotic nematic mesophase from $68.5^{\circ} \mathrm{C}$ to $112^{\circ} \mathrm{C}$, from which temperature on it is present as an isotropic liquid. ${ }^{24}$ The feature in Faraday rotation is present for the crystalline phase. In the discotic nematic phase the feature shifts to the blue, and finally the band in Faraday rotation is no longer visible in the isotropic liquid phase. This shift from crystalline to isotropic liquid is accompanied by a change in conformation and conformational freedom. In the crystalline phase the molecule is present in a fairly rigid, propeller geometry. ${ }^{45}$ The disappearance of the Faraday rotation band in the isotropic liquid goes along with the complete loss of this conformational refinement for both, TTB and TTB*. Consistent with this, a diluted solution in dichloromethane does not display a band in the Faraday rotation.

\section{Conclusion}

Materials with high Verdet constants and low absorption in the spectral region of interest are particularly interesting for a wide variety of applications of Faraday rotation. In search of these properties, we have characterized the Verdet constant of an unexplored class of organic molecules. A Verdet constant of almost 2.5. $10^{5} \mathrm{deg} \mathrm{T}^{-1} \mathrm{~m}^{-1}$ is found with no associated absorption, three orders of magnitude larger than the largest known for an organic molecule in a region without spectral features. Based on the available data, the enormous Faraday rotation is potentially caused by a combination of intense low-energy singlet excitations close to $520 \mathrm{~nm}$ and further resonant enhancement by a triplet excitation which is calculated to be right in the relevant wavelength window. While the transition is formally spin-forbidden, due to some degree of spin-orbit coupling it may provide a mechanism for enhancing the Faraday rotation. We plan to address the cause of the strong Faraday rotation around $520 \mathrm{~nm}$ in more detail in a follow-up study. We are able to switch the Faraday rotation by changing the particular phase of the molecule. These results demonstrate a new class of Faraday rotating materials with great potential to replace current materials and in- 
crease the sensitivity in applications. The inherent flexibility in the synthesis, aggregation state and concentration of this class of molecules opens a new field of research in Faraday rotation.

\section{Supporting Information Available}

Calculated spectra and triplet excitations with a large spin-free component of transition dipole for TTB and BTB* model molecules. Experimental NMR spectra for BTB*. This material is available free of charge via the Internet at http://pubs.acs.org/.

\section{Acknowledgement}

SV is grateful for the financial support from the FWO-Vlaanderen. SVC and PW received funding from the Agency for Innovation by Science and Technology (IWT) Flanders (research funded by a PhD grant of the Agency for Innovation by Science and Technology (IWT)). We are grateful to the University of Leuven (GOA) for financial support. We would like to thank the Research fund of the KU Leuven. We are grateful to the Research Foundation Flanders (FWO) for financial support. VKV acknowledges financial support from the EPSRC grant EP/G060649/1 and the VS.040.10N grant from the BAS and the FWO. MS acknowledges financial support from the Foundation for Polish Science ('START'). JA acknowledges support by the National Science Foundation (CHE0952251) and the Center for Computational Research at UB. GH acknowledges financial support by the Spanish government through project CTQ2010-18813. We acknowledge useful discussions with M. Bloemen, W. Brullot and M. Vanbel.

\section{References}

(1) Faraday, M. Experimental Researches in Electricity; R. Taylor \& W. Francis, 1839-1855; Vol. III.

(2) Takeda, H.; John, S. Phys. Rev. A 2008, 78, 023804. 
(3) Budker, D.; Kimball, D. F.; Rochester, S. M.; Yashchuk, V. V.; Zolotorev, M. Phys. Rev. A 2000, 62, 043403.

(4) Bohnert, K.; Gabus, P.; Kostovic, J.; Brändle, H. Op. Laser. Eng. 2005, 43, 511-526.

(5) Bera, S. C.; Chakraborty, S. Measurement 2011, 44, 1747-1752.

(6) Dötsch, H.; Bahlmann, N.; Zhuromskyy, O.; Hammer, M.; Wilkens, L.; Gerhardt, R.; Hertel, P.; Popkov, A. F. J. Opt. Soc. Am. B 2005, 22, 240-253.

(7) Bullmore, E.; Sporns, O. Nat. Rev. Neurosci. 2009, 10, 186-198.

(8) Lvovsky, A. I.; Sanders, B. C.; Tittel, W. Nat. Photon. 2009, 3, 706-714.

(9) Tepper, T.; Ilievski, F.; Ross, C. A.; Zaman, T. R.; Ram, R. J.; Sung, S. Y.; Stadler, B. J. H. J. Appl. Phys. May 2003, 93, 6948-6950.

(10) Hansen, P.; Witter, K.; Tolksdorf, W. Phys. Rev. B. 1983, 27.

(11) Huang, M.; Zhang, S. Y. Appl. Phys. A.-Mater 2002, 74, 177-180.

(12) Abulafya, G.; Le Gall, H. Solid State Commun. 1972, 11, 629-633.

(13) Dalton, L. R. Pure Appl. Chem. 2004, 76, 1421-1433.

(14) Jaszunski, M.; Jørgensen, P.; Rizzo, A.; Ruud, K.; Helgaker, T. Chem. Phys. Lett. 1994, 222, 263-266.

(15) Botek, E.; Champagne, B.; Verbiest, T.; Gangopadhyay, P.; Persoons, A. ChemPhysChem 2006, 7, 1654-1656.

(16) Isai, K.; Suwa, M.; Watarai, H. Anal. Sci. 2009, 25, 1-3.

(17) Vandendriessche, S.; Valev, V. K.; Verbiest, T. Phys. Chem. Chem. Phys. 2012, 14, 18601864. 
(18) Suwa, M.; Miyamoto, K.; Watarai, H. Anal. Sci. 2013, 29, 113-119.

(19) Gangopadhyay, P.; Voorakaranam, R.; Lopez-Santiago, A.; Foerier, S.; Thomas, J.; Norwood, R. A.; Persoons, A.; Peyghambarian, N. J. Phys. Chem. C 2008, 112, 8032-8037.

(20) Kimura, M.; Kondo, H.; Hattori, S. J. Phys. Soc. Jpn 1965, 20, 1778-1782.

(21) Eberhardt, W. H.; Renner, H. J. Mol. Spectrosc. 1961, 6, 483-491.

(22) Snowden Jr., B. S.; Eberhardt, W. H. J. Mol. Spectrosc. 1965, 18, 372-383.

(23) Brown, S. H.; Chiu, Y.-N. J. Chem. Phys. 1978, 69, 3579-3588.

(24) Hennrich, G.; Omenat, A.; Asselberghs, I.; Foerier, S.; Clays, K.; Verbiest, T.; Serrano, J. Angew. Chem. Int. Ed. 2006, 45, 4203-4206.

(25) de Vega, L.; van Cleuvenbergen, S.; Depotter, G.; García-Frutos, E. M.; Gómez-Lor, B.; Omenat, A.; Tejedor, R. M.; Serrano, J.; Hennrich, G.; Clays, K. J. Org. Chem. 2012, 77, 10891-10896.

(26) Valev, V. K.; Wouters, J.; Verbiest, T. Am. J. Phys. 2008, 76, 626-629.

(27) TURBOMOLE V5.7.1 2005, Quantum Chemistry Group, University of Karlsruhe, Germany.

(28) Ahlrichs, R.; Bär, M.; Häser, M.; Horn, H.; Kölmel, C. Chem. Phys. Lett. 1989, 162, 165-169.

(29) Becke, A. D. Phys. Rev. A. 1988, 38, 3098-3100.

(30) Perdew, J. P. Phys. Rev. B. 1986, 33, 8822-8824.

(31) Perdew, J. P. Phys. Rev. B. 1986, 34, 7406-7406.

(32) Weigend, F.; Ahlrichs, R. Phys. Chem. Chem. Phys. 2005, 7, 3297-3305.

(33) Becke, A. D. J. Chem. Phys. 1993, 98, 5648-5652.

(34) Lee, C.; Yang, W.; Parr, R. G. Phys. Rev. B. 1988, 37, 785-789. 
(35) Stephens, P. J.; Devlin, F. J.; Chabalowski, C. F.; Frisch, M. J. J. Chem. Phys. 1994, 98, $11623-11627$.

(36) Peach, M. J. G.; Williamson, M. J.; Tozer, D. J. J. Chem. Theory Comput. 2011, 7, 35783585.

(37) Tamm, I. J.Phys.(USSR) 1945, 9, 449.

(38) Dancoff, S. M. Phys. Rev. 1950, 78, 382-385.

(39) Coriani, S.; Hättig, C.; Jørgensen, P.; Halkier, A.; Rizzo, A. Chem. Phys. Lett. 1997, 281, $445-451$.

(40) Banerjee, A.; Autschbach, J.; Ziegler, T. Int. J. Quantum Chem. 2005, 101, 572-578.

(41) Krykunov, M.; Banerjee, A.; Ziegler, T.; Autschbach, J. J. Chem. Phys. 2005, 122, 074105.

(42) Oliva, M. M.; Casado, J.; López Navarrete, J. T.; Hennrich, G.; van Cleuvenbergen, S.; Asselberghs, I.; Clays, K.; Ruiz Delgado, M. C.; Brédas, J.-L.; Seixas de Melo, J. S.; De Cola, L. Chem.-Eur. J. 2009, 15, 8223-8234.

(43) Van Cleuvenbergen, S.; Hennrich, G.; Willot, P.; Koeckelberghs, G.; Clays, K.; Verbiest, T.; van der Veen, M. A. J. Phys. Chem. C 2012, 116, 12219-12225.

(44) Ho, V.; Boudouris, B. W.; Segalman, R. A. Macromolecules 2010, 43, 7895-7899.

(45) Hennrich, G.; Tejedor, R.; Serrano, J.; Ramirez, F. Submitted to Langmuir 2013, 


\section{Graphical TOC Entry}

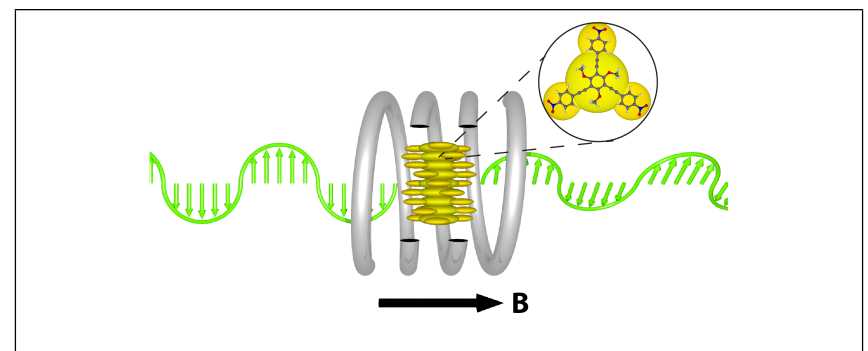

